\title{
ANALYSIS OF NEUTRON FIELDS GENERATED IN SPALLATION TARGETS OF B-URAN EXPERIMENTAL ASSEMBLY USING MONTE CARLO METHOD
}

\author{
Ondřej ŠŤASTnÝ ${ }^{a, b, *}$, Miroslav Zeman ${ }^{a, d}$, DuŠAn KRÁL ${ }^{a}$, \\ Karel KatovskÝ ${ }^{a}$, Elmira Melyan ${ }^{a, c}$, Jindřich $\operatorname{ADAm}^{a, d}$, \\ Alexander Alexandrovich Solnyshikin ${ }^{d}$ \\ ${ }^{a}$ Brno University of Technology, Faculty of Electrical Engineering and Communication, Department of Electrical \\ Power Engineering, Technická 3082/12, 61600 Brno, Czech Republic \\ ${ }^{b}$ State Office for Nuclear Safety, Department of Non-Proliferation, Senovážné nám. 9, 11000 Prague 1, Czech \\ Republic \\ ${ }^{c}$ Yerevan State University, Faculty of Physics, 1 Alex Manoogian Street, 0025 Yerevan, Armenia \\ $d$ Joint Institute for Nuclear Research, Laboratory of Nuclear Problems, Joliot-Curie 6, 141980 Dubna, Russia \\ * corresponding author: xstast30@stud.feec.vutbr.cz
}

\begin{abstract}
The aim of this paper is to introduce experimental assembly B-URAN and the results of Monte Carlo simulations of neutron fields, which will be generated by using various spallation targets. This experimental assembly was constructed in Joint Institute of Nuclear Research in Dubna, Russian Federation, in order to study accelerator driven systems fundamental characteristics. Beam of 660 $\mathrm{MeV}$ protons should be used for that purpose. The MCNP model of such set-up has been developed at Brno University of Technology, Czech Republic. The goal is to get data needed for prediction of reaction rates in detectors placed in B-URAN experimental channels. Such data will be experimentally validated later. Furthermore, simulations of radiation exposure around this experimental assembly were performed.
\end{abstract}

Keywords: Accelerator driven systems, B-URAN, Monte Carlo simulations, reaction rates, spallation reactions.

\section{INTRODUCTION}

There is an area of nuclear power production, which is still considered as its weakness - spent nuclear fuel, which contains a lot of highly radioactive fission products and long-lived actinides; therefore, it has to stay separated from the environment forever. Deep geological repositories can serve as the most conservative solution for this purpose. Nevertheless, there are other options, such as reprocessing, which provides possibility to use plutonium again as a part of MOX fuel. However, fission products and long-lived actinides remain as a heritage of nuclear power production and they are treated as radioactive waste.

Several decades ago, one promising solution was proposed - Accelerator Driven Systems (ADS) [1, which have the potential to "incinerate" such kind of problematic isotopes and therefore minimize the amount of radioactive waste after reprocessing the spent nuclear fuel. ADS usually consist of accelerator, spallation target and blanket. Accelerator produces accelerated particles (protons, deuterons) to high energies (hundreds of $\mathrm{MeV}$ or ones of $\mathrm{GeV}$ ) which than trigger spallation reactions in target material. Spallation reactions produce high-energy neutron fields around the target that can be used to transmute ("incinerate") radionuclides located in surrounding blanket (see the example at Figure 11 neutron yield by using $800 \mathrm{MeV}$ protons and tungsten target).

There has been a lot of research activities worldwide focused on this area so far, but still the compact models and nuclear data libraries describing the parameters of generated neutron fields are missing (for more information see [3 6 ).

One of the research facilities with long experience in this research is Joint Institute of Nuclear Research (JINR) in Dubna, Russian Federation. Group E+T RAW (Energy \& Transmutation of Radioactive Waste) with experimental assembly QUINTA 3 can be mentioned in this regard. Local accelerator Phasotron produces particles with energies up to $660 \mathrm{MeV}$ and various targets are available, as well. This creates a great opportunity for many researches for studying spallation reactions and parameters of generated neutron fields.

\section{DESCRIPTION OF PROPOSED EXPERIMENT WITH B-URAN EXPERIMENTAL ASSEMBLY}

\subsection{B-URAN EXPERIMENTAL ASSEMBLY}

Latest experimental assembly B-URAN was designed at JINR for studies of spallation neutrons behavior in quasi-infinite geometry. This experimental assembly is a cylinder made from depleted uranium with diameter 


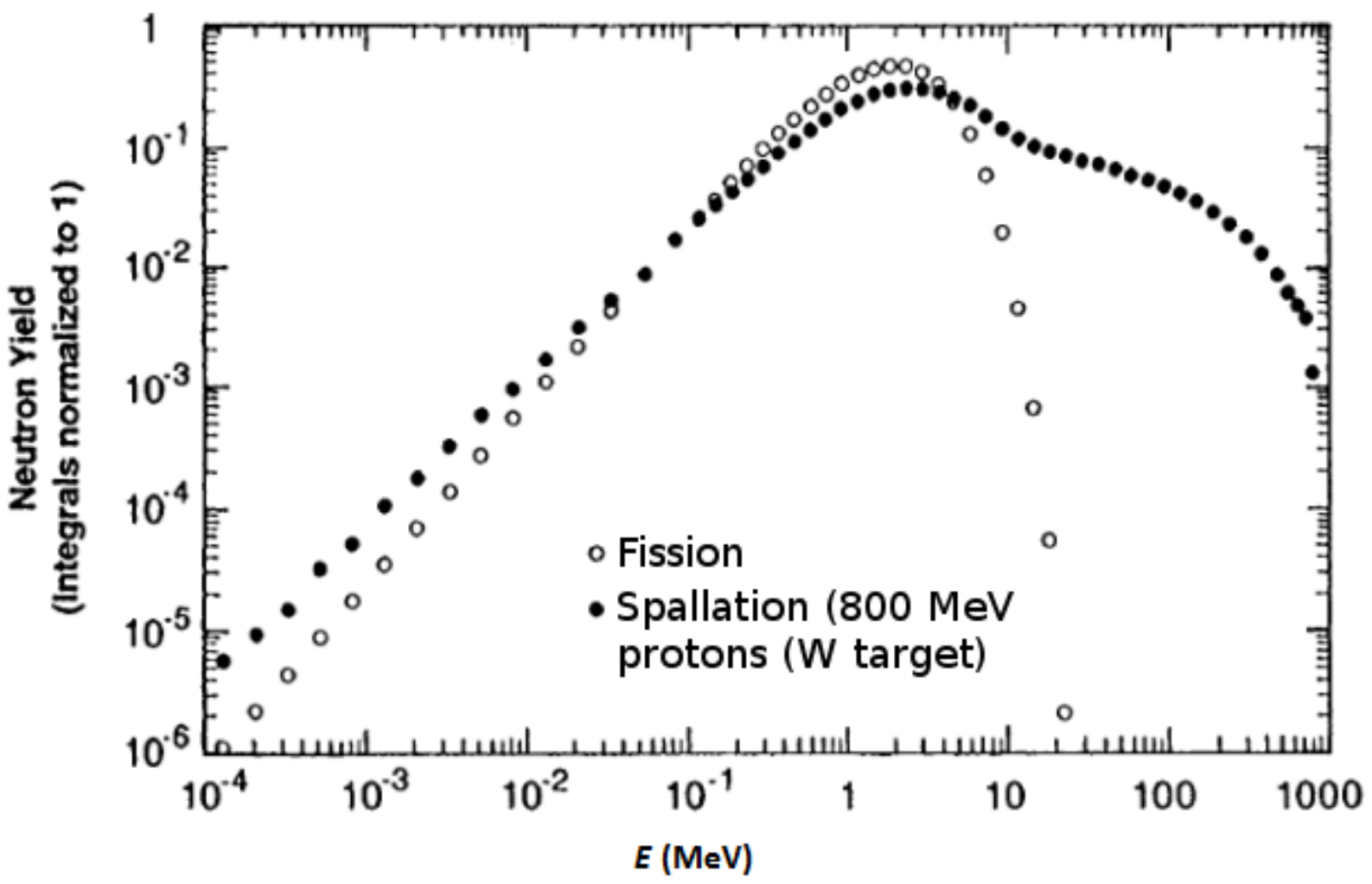

Figure 1. Neutron Yield from fission and spallation inside tungsten target [2]

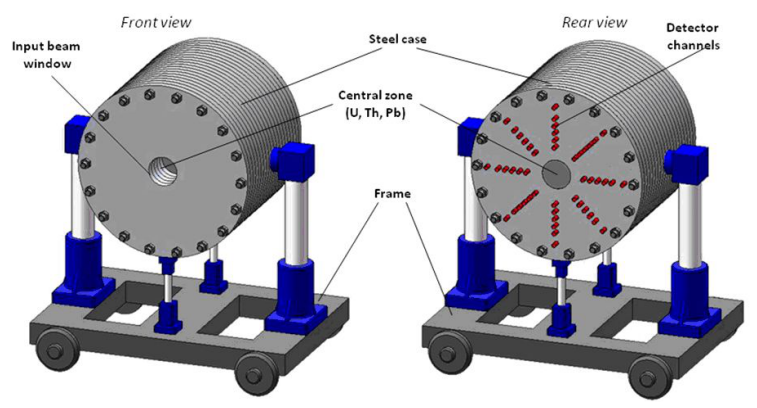

FiguRE 2. Schematic drawings of B-URAN, front and rear view 7

of $120 \mathrm{~cm}$ and length of $100 \mathrm{~cm}$. It is coated with 10 $\mathrm{cm}$ thick steel layer and weights app. 20 tons. Central zone allows positioning replaceable cylindrical targets with $20 \mathrm{~cm}$ in diameter. For experimental purposes, B-URAN offers large amount of axial experimental channels 3. More details are shown in Figure 2

\subsection{Spallation targets}

Several types of spallation target were considered lead, graphite and bismuth. Lead target was $100 \mathrm{~cm}$ in length, but due to the high leakage of neutrons from the front part of B-URAN, it was decided to make a hole $8 \mathrm{~cm}$ in diameter and $40 \mathrm{~cm}$ in length so the particle beam could penetrate deeper into the target geometry in order to have more convenient distribution of the generated neutron field inside the inner

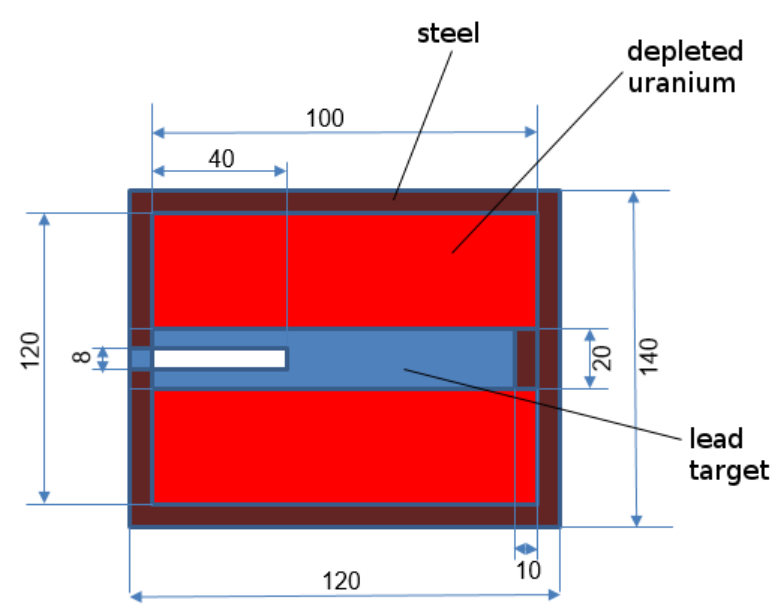

FiguRE 3. B-URAN sectional view, lead target 8

part of B-URAN. For further details, see Figure 3

\subsection{MCNP MODEL OF THE PROPOSED EXPERIMENT}

First, the MCNP 6.2 9] model of B-URAN experimental assembly was developed. It was decided to simplify it in order to decrease the CPU time needed, therefore, only one plane of experimental channels (9 in total) was considered. Each experimental channel is $3 \mathrm{~cm}$ in diameter, and they are located at positions 14 , $18,22,26,30,34,38,44$ and $52 \mathrm{~cm}$ from the B-URAN axis. The source of particles is a proton beam of en- 


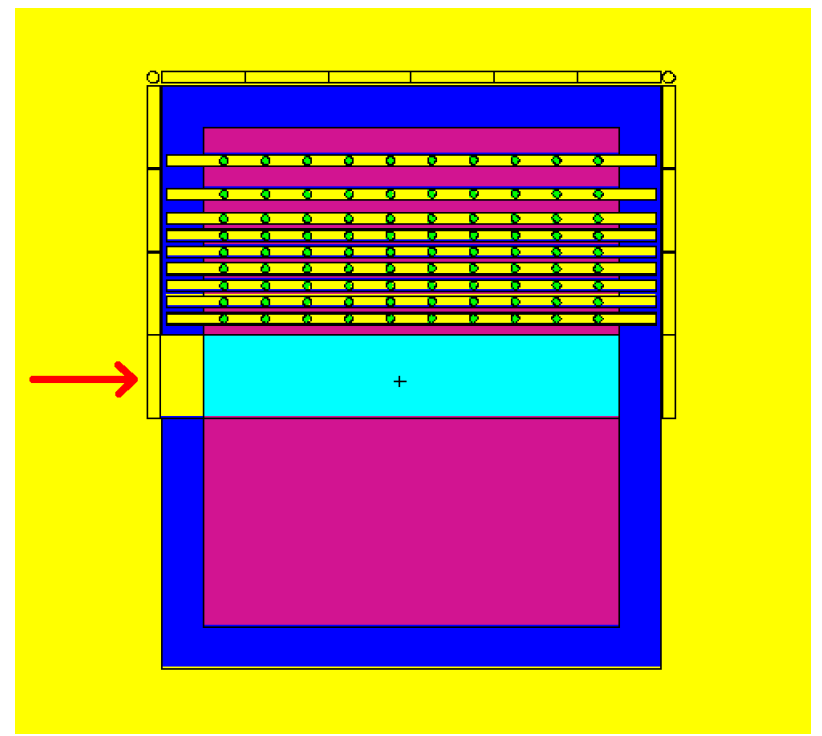

FigURE 4. B-URAN sectional view, graphite target, red arrow is a symbol of proton beam (created by MCNP)

ergy $660 \mathrm{MeV}$ impacting on target in B-URAN central zone. For neutron spectra measurement a set of 10 threshold activation detectors is placed inside each considered experimental channel along the B-URAN's length. Preliminary, it was decided that small "coin" detectors (diameter $2 \mathrm{~cm}$, height $0.5 \mathrm{~cm}$ ) made from bismuth will be used. The aim is to detect $(n, x n)$ reaction rates up to several hundreds of $\mathrm{MeV}$. According to the results of calculation, different materials can be used for this purpose in further research.

See the Figure 4 for detailed image of B-URAN experimental assembly and location of threshold activation detectors inside experimental channels. It should be stated, that the B-URAN experimental assembly is located at story with height of $250 \mathrm{~cm}$, so there is $45 \mathrm{~cm}$ of space between the ceiling and the top of concrete shielding. Both ceiling and the floor are made from $1 \mathrm{~cm}$ thick steel plate. All these details correspond with the real conditions at JINR.

Surrounding environment of B-URAN was represented by air in shape of cylinder with radius 200 $\mathrm{cm}$. There were also additional "control volumes" filled with air around B-URAN which were used for monitoring of neutron leakage from the experimental assembly.

Graphite target was $100 \mathrm{~cm}$ in length, despite bismuth target was $50 \mathrm{~cm}$ only and was placed in the rear position inside B-URAN target channel - see Figure 4.

For neutron and gamma dose calculation, a simplified model of B-URAN has been created. It also involved its surroundings (concrete shielding) at the story, where this experimental assembly is located.

All calculations were performed using default physics settings of MCNP (model CEM03.03 [10]) and photofission settings was turned on. Concerning used nuclear data libraries, see Table 1. For thermal treating a free-gas model was involved, no S(a,b) li-

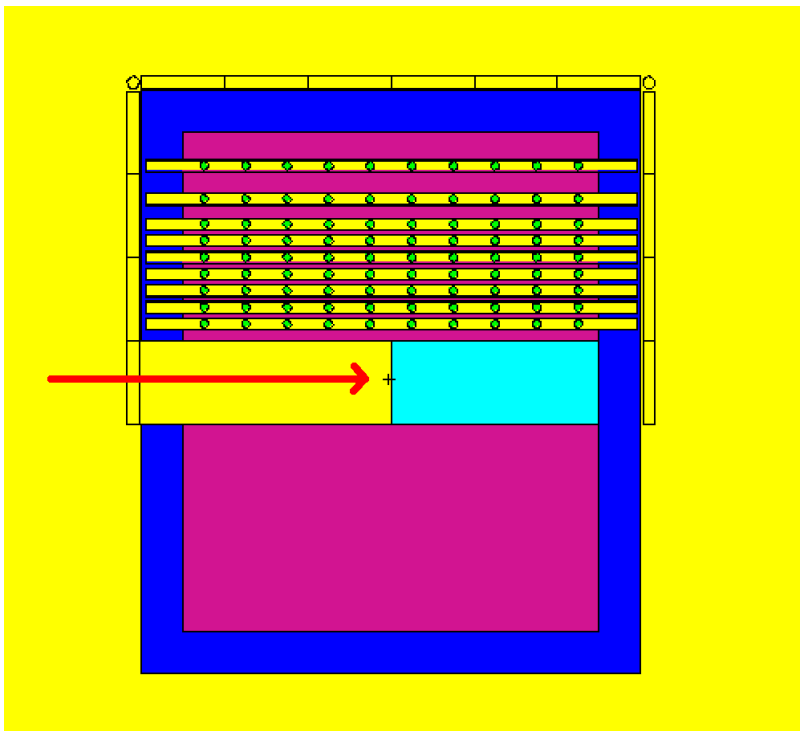

FiguRE 5. B-URAN sectional view, bismuth target, red arrow is a symbol of proton beam (created by MCNP)

braries were used. For all other reactions, internal default MCNP models were used.

\subsection{EXPECTED MODEL OUTCOMES}

The main aim of the model was to determine neutron flux values inside the threshold activation detectors (tally F4 - flux) in order to get data for further reaction rates calculation. Subsequently, a visual information about several types of particles spatial distribution (mesh tally, CMESH type 1 - flux) was also seek. Finally, rough estimation of radiation dose around B-URAN during irradiation was investigated (mesh tally, RMESH type 1 - dose), as well.

\section{Results AND Discussion}

Results of neutron flux calculation for threshold activation detectors can be found in Figure 6. Average relative error of values for detectors using spallation target made from graphite, lead and bismuth are $0.17 \%-0.6 \%-1.08 \%$. It clearly shows that graphite target provides several orders higher values than other target materials (lead, bismuth) - this needs to be evaluated by comparison with experimental results. From the neutron yield point of view, graphite target seems to be the best option. However, other features of target materials should be taken into consideration, such as thermal stability, which could be in favor for heavy metals such as lead.

Original idea was to perform these calculations for targets made from depleted uranium and thorium as well. However, it turned out, that these heavy metals with fast fission factor need longer computation times at ordinary workstation.

Furthermore, neutron flux mesh was calculated for several targets - graphite, lead and bismuth and results of the neutron flux from various materials is 


\begin{tabular}{cl}
\hline Library & Materials \\
\hline ENDF/B-7.1 & nat. $\mathrm{C},{ }^{14} \mathrm{~N},{ }^{16} \mathrm{O},{ }^{55} \mathrm{Mn},{ }^{209} \mathrm{Bi},{ }^{234} \mathrm{U},{ }^{235} \mathrm{U},{ }^{238} \mathrm{U}$ \\
\hline ENDL-85 & nat. $\mathrm{Ar}$ \\
\hline ENDF/B-5 & nat. Cr, nat. Ni, nat. Pb \\
\hline ENDF/B-5 T2 group upgrade [1] & nat. Fe \\
\hline MCPLIB-v.04 from ENDF/B-5 (photon data) & $\begin{array}{l}\text { nat. C, nat. N, nat. O, nat. Ar, nat. Cr, }{ }^{55} \mathrm{Mn}, \text { nat. Fe, } \\
\text { nat. Ni, nat. Pb, }{ }^{209} \mathrm{Bi}, \text { nat. U }\end{array}$ \\
\hline ENDF/B-7.0 (photonuclear data) & ${ }^{14} \mathrm{~N},{ }^{55} \mathrm{Mn},{ }^{209} \mathrm{Bi},{ }^{235} \mathrm{U},{ }^{238} \mathrm{U}$ \\
\hline LA150 (photonuclear data) & ${ }^{16} \mathrm{O}$ \\
\hline ENDF/B-7.0 (proton data) & ${ }^{14} \mathrm{~N},{ }^{16} \mathrm{O},{ }^{209} \mathrm{Bi}$ \\
\hline EL03 (electron data) [12] & nat. C, nat. N, nat. O, nat. Ar, nat. Cr, ${ }^{55} \mathrm{Mn}$, nat. Fe, \\
& nat. Ni, nat. Pb, ${ }^{209} \mathrm{Bi}$, nat. U \\
\hline
\end{tabular}

TABLE 1. Nuclear data libraries for MCNP calculations

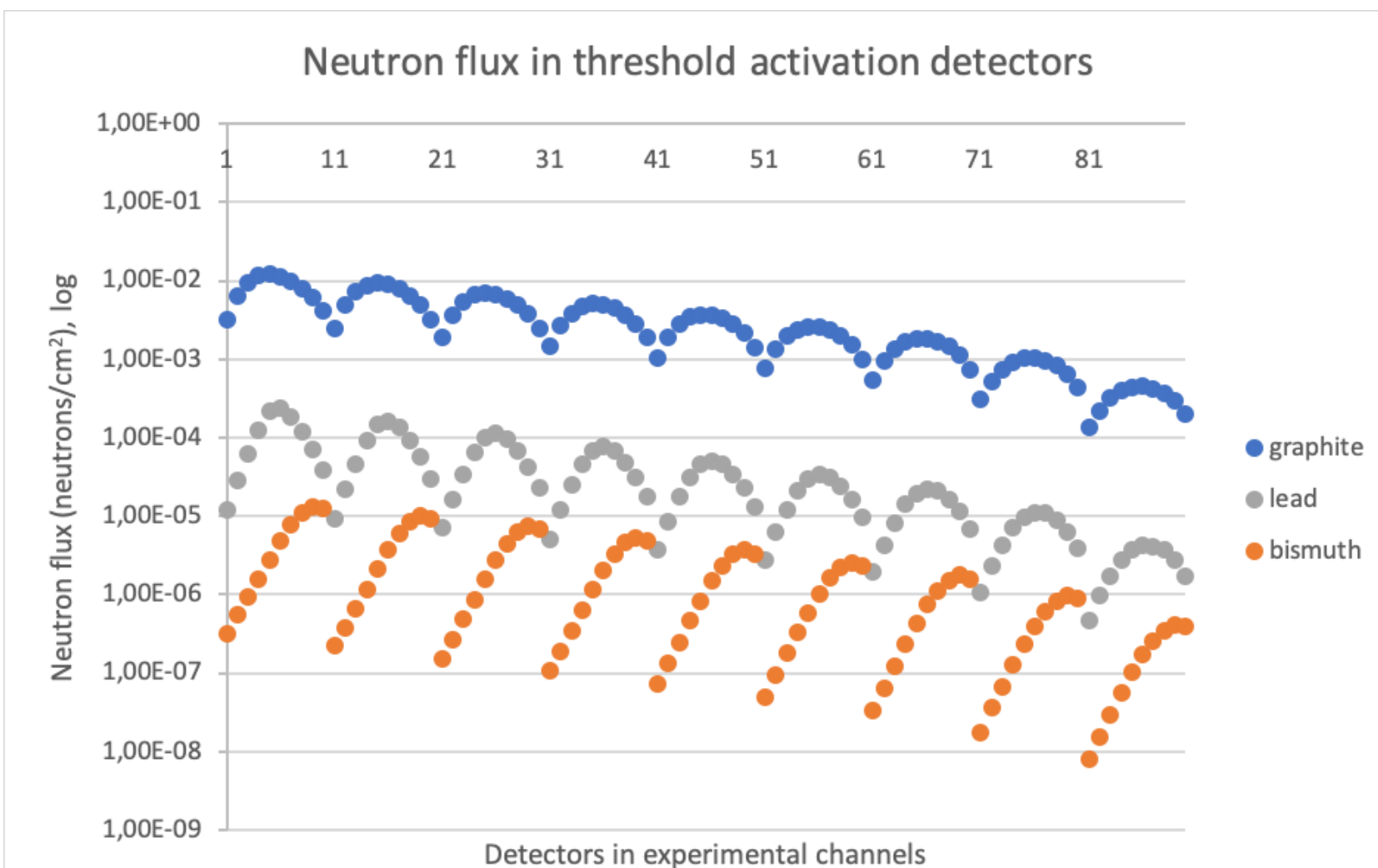

FIGURE 6. Comparison of several targets, neutron flux (neutrons $/ \mathrm{cm}^{2}$ ), 9 sets of detectors in experimental channels (calculated by MCNP)

possible to see the in Figure 7, where is their comparison. These results fully corresponds with graph in Figure 6. Since the bismuth target was placed in the rear position of the B-URAN target channel, the maximum values of neutron flux were located at the end of each experimental channel. In case of lead target, which fills the whole target channel (with hole in the center), the shape of neutron flux graph almost equals to normal distribution. For graphite target, the maximum of neutron flux in each channel can be found at detectors in the middle of B-URAN length.

Moreover, radiation dose around B-URAN during irradiation was computed, as well. Drawing of the B-URAN surroundings including concrete shielding is shown in Figure 8. Results can be found in Figure 9 - neutron and gamma dose. Influence of concrete shielding located in front of B-URAN is clearly visible as well as a small tunnel which leads the particle beam to target.

The different behavior of neutron and gamma ra- 


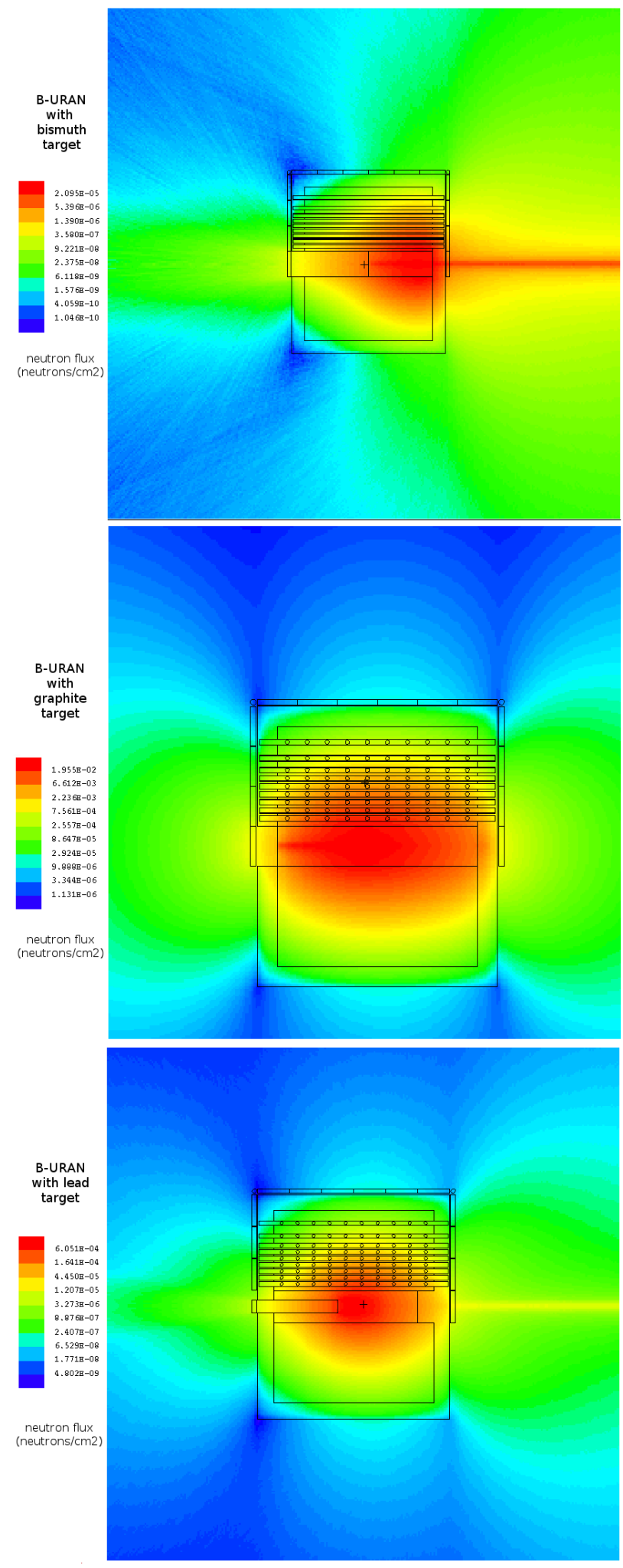

FiguRE 7. Comparison of neutron flux mesh of several targets (created by MCNP)

diation is clearly visible. This particular concrete shielding provides very efficient protection against gamma radiation, unlike neutron radiation still creates quite compact neutron field behind the shielding, which decreases by three to five orders of magnitude only, probably because of neutron transport through

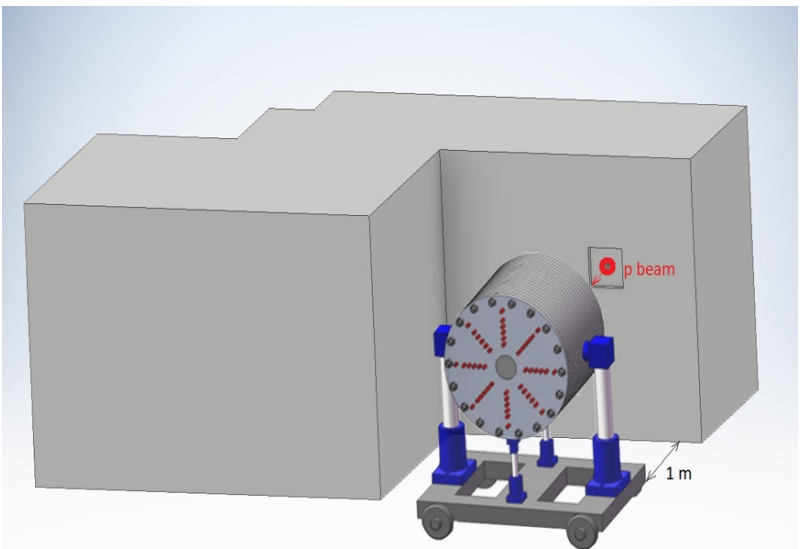

FIGURE 8. B-URAN surroundings including concrete shielding [8]

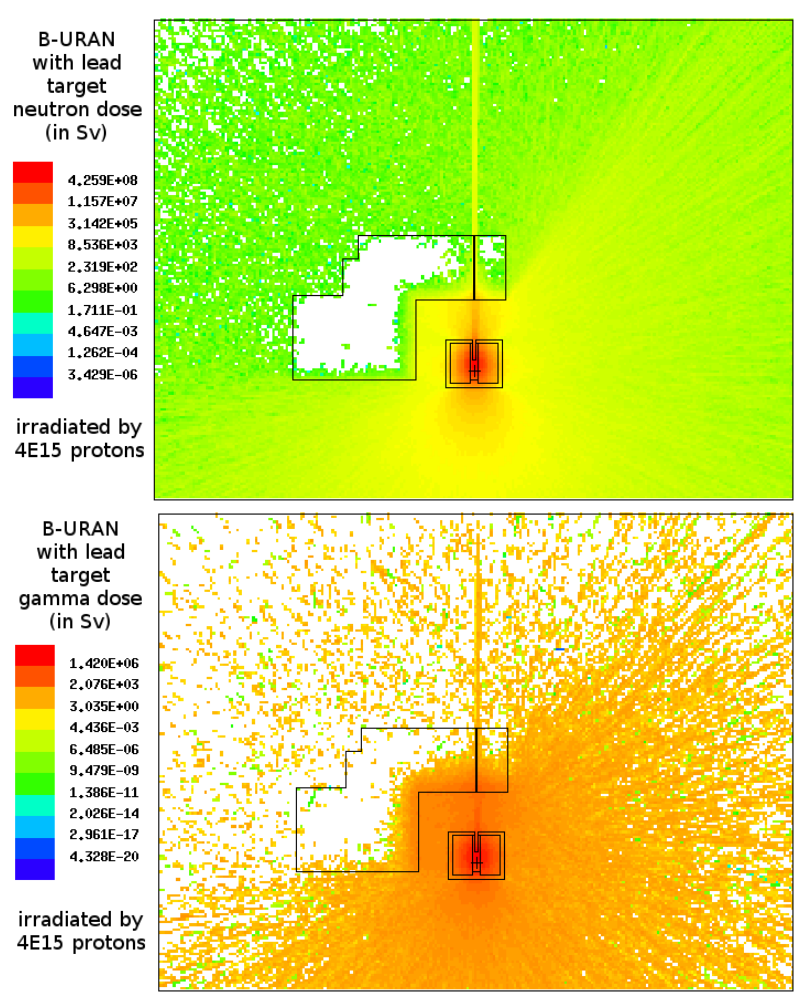

Figure 9. Neutron and gamma dose around B-URAN experimental assembly with lead target, at level of proton beam, irradiated by $4 \mathrm{E} 15$ protons (created by $\mathrm{MCNP}$ )

the space between the top of the shielding and the ceiling.

\section{Conclusions}

In this paper, the neutron flux for bismuth threshold activation detectors in experimental channels of B-URAN assembly was calculated. These values can be then used for reaction rates determination. Furthermore, several targets (graphite, lead and bismuth) were taken into consideration and neutron flux mesh was calculated for them, as well.

Based on values for neutron flux in threshold acti- 
vation detectors, it can be stated, that graphite target provides several orders higher values than other target materials - this needs to be validated by comparison with experimental results. Other question is graphite thermal stability, which could be in favor for heavy metals such as lead.

Since the targets made from depleted uranium and thorium were proposed as well, the same calculations should be also performed for them in further research. Moreover, geometrical properties of targets should be also considered. The aim should be to minimize neutron leakage from the front and rear part of BURAN assembly and to maximize neutron field usage inside the threshold activation detectors located in experimental channels. As a result, the aim for further research in this area should be to propose a target design combining light and heavy materials together.

Based on the gained knowledge, most efficient material composition of detectors should be investigated. For purposes of this article, only single material (bismuth) has been considered. Considering neutron and gamma dose calculations, the radiation dose around B-URAN has been estimated. According to obtained values, which have been calculated for typical irradiation session at JINR Phasotron $\left(4 \times 10^{15}\right.$ protons), the doses from gamma and neutrons could be very high (see Figure 9). Following to that, radiation protection measures have to be clearly defined and regularly verified. Moreover, the influence of neutron radiation to the concrete shielding should be investigated (especially with regard to possible concrete activation by neutrons).

\section{ACKNOWLEDGEMENTS}

Part of this research work has been carried out in the Centre for Research and Utilization of Renewable Energy (CVVOZE: http://www.cvvoze.cz) and part within Energy and Transmutation of Radioactive Waste collaboration (E\&T RAW: http://et.jinr.ru).

Authors gratefully acknowledge financial support from the Ministry of Education, Youth and Sports of the Czech Republic under Brno University of Technology specific research programme (project No. FEKT-S-17-4784 "New Technologies for sustainable power engineering") and under international research cooperative programme of JINR Dubna and the Czech Republic (projects of joint $3+3$ committee No. 11/159/2018 "Neutron spectral characterization using spectral shield analysis" and 12/159/2018 "Study of neutron flux in spallation targets").

\section{REFERENCES}

[1] C. Bowman, E. Arthur, P. Lisowski, et al. Nuclear energy generation and waste transmutation using an accelerator-driven intense thermal neutron source. Nuclear Inst and Methods in Physics Research pp. 336-367, 1992. DOI:10.1016/0168-9002(92)90795-6
[2] D. Král. Investigation of thorium utilization in accelerator driven nuclear reactors. Master's thesis, Brno University of Technology, Brno 2017.

[3] W. Furman, J. Adam, L. Závorka, et al. Recent results of the study of ADS with $500 \mathrm{~kg}$ natural uranium target assembly QUINTA irradiated by deuterons with energies from 1 to $8 \mathrm{GeV}$ at JINR NUCLOTRON. Proceedings of Science 2012. http: //www.sujv.cz/cz/index .php?Ns=202\&id=1000085

[4] A. Koning, D. Rochman. Modern Nuclear Data Evaluation With The TALYS Code System. Nuclear Data Sheets 113 2012. http://www.sciencedirect. com/science/article/pii/S0090375212000889.

[5] D. Rochman, A. Koning, J. Sublet, et al. The TENDL library: hope, reality and future. Proceedings of the International Conference on Nuclear Data for Science and Technology 2016. Bruges, Belgium, https://tendl. web.psi.ch/bib_rochman/tendl.nd2016.pdf

[6] J. Thomason. The ISIS Spallation Neutron and Muon Source - The first thirty-three years. Nuclear Instruments and Methods in Physics Research Section A: Accelerators, Spectrometers, Detectors and Associated Equipment 917:61-67, 2019. ISSN 0168-9002, DOI:10.1016/j.nima.2018.11.129

[7] JINR. Energy and Transmutation of RadioActive Waste [online], Dubna, Moscow region, Russian Federation, 2019 [quote: 2019-05-26], http://et.jinr.ru/pict/pic_buran.jpg

[8] P. Tichý, J. Adam, A. Baldin, et al. Experimental investigation and Monte Carlo simulations of radionuclide production inside the Uranium spallation target QUINTA irradiated with a $660 \mathrm{-MeV}$ proton beam. EPJ Web Conf 204040032019. DOI:10.1051/epjconf/201920404003.

[9] C. Werner. MCNP Users Manual - Code Version 6.2. Los Alamos National Laboratory, report LA-UR-17-29981 2017.

[10] CEM03.03. Monte-Carlo Code system to calculate nuclear reactions in the framework of the improved cascade-exciton model. Stepan Mashnik and Arnold Sierk: CEM0303 User Manual, Los Alamos National Laboratory, Report LA-UR-12-01364 2012. http://www oecd-nea.org/tools/abstract/detail/psr-0532/

[11] E. Arthur, P. Young. Evaluated neutron-induced cross sections for $54,56 \mathrm{Fe}$ to $40 \mathrm{MeV}$. Los Alamos National Laboratory report No LA-8626-MS 1980.

[12] K. Adams. Electron Upgrade for MCNP4B. Los Alamos National Laboratory internal memorandum, $X-5-R N(U)-00-142000$. 